\title{
Does socio-economic status explain the differentials in malaria parasite prevalence? Evidence from The Gambia
}

Sheriff T Sonko ${ }^{* *}$, Malanding Jaiteh ${ }^{2}$, James Jafali ${ }^{3}$ Lamin BS Jarju', Umberto D'Alessandro ${ }^{3}$, Abu Camara ${ }^{4}$, Musu Komma-Bah ${ }^{5}$ and Alieu Saho ${ }^{5}$

\begin{abstract}
Background: Malaria is commonly associated with poverty. Macro-level estimates show strong links between malaria and poverty, and increasing evidence suggests that the causal link between malaria and poverty runs in both directions. However, micro-level (household and population) analyses on the linkages between malaria and poverty have often produced mixed results.

Methods: The Gambia Malaria Indicator Survey (MIS) 2010/11 was carried out between November 2010 and January 2011. Laboratory-confirmed malaria and wealth quintiles were used to assess the association of socio-economic status and malaria infection in children and the general population. Simple and multiple logistic regressions and survey data analysis procedures, including linearized standard errors to account for cluster sampling and unequal selection probabilities were applied.
\end{abstract}

Results: Children (six to 59 months) from the second, third, fourth and richest quintiles were significantly less likely to have malaria compared to children from the poorest quintiles. Children (five to 14 years) from the fourth and richest quintiles were also significantly less likely to have malaria compared to those from the poorest quintiles. The malaria burden has shifted from the under-five children (six to 59 months) to children aged five to 14 years. Malaria prevalence was significantly higher in the Central River Region compared to the Upper River Region; and males bear the malaria brunt more than females. Children (six to 59 months) and children (five to 14 years) living in houses with poor walls, floors, roofs and windows were significant associated with higher prevalence of malaria. However, in the general population, only poor wall housing materials were associated with higher prevalence of malaria.

Conclusions: Investments in strategies that address socio-economic disparities and improvements in the quality of housing could, in the long term, significantly reduce the malaria burden in the poorest communities.

Keywords: Malaria, The Gambia, Poorest, Poverty, Socio-economic status

\section{Background}

The worldwide malaria burden is currently estimated at about 207 million cases and 627,000 deaths. Sub-Saharan Africa bears the heaviest brunt, with $90 \%$ of all deaths, $77 \%$ among children under-five. Nevertheless, between 2000 and 2012, malaria mortality rates have decreased by $42 \%$ worldwide (by $48 \%$ in children under-five) and by $49 \%$ in the African Region (by $54 \%$ in children under-five) [1].

\footnotetext{
* Correspondence: stsonko@outlook.com

${ }^{1}$ National Malaria Control Programme, Kanifing Institutional Layout, Plot \# 17, Kanifing, The Gambia

Full list of author information is available at the end of the article
}

Malaria is commonly associated with poverty [2-10]. Macro-level estimates show strong links between malaria and poverty. For instance, the malaria burden is highest in the poorest countries, particularly in sub-Saharan Africa, where poverty is widespread and with little economic growth over the past quarter century $[5,6]$. The gross national product (GNP) in malaria endemic countries is more than half lower than in non-endemic countries [6]. The poorest $20 \%$ of the world's population contribute to $58 \%$ of all malaria deaths [11]. Increasing evidence at macro-level suggests that the causal link between malaria and poverty runs in both directions [3-6]. 
A review of micro-level (household and population) analyses on the link between malaria and poverty has produced mixed results [12]. Out of nine studies, only two reported a significant association between malaria and poverty [12]. Data from 29 demographic and health surveys (DHS) in 22 countries were used for an aggregatelevel regional data analysis for West and Central Africa, and East and Southern Africa, including individual child and country-by-country analysis. No differences were found at the household level in the incidence of fever between the poor and the less poor, though significant differences were found at more aggregate-levels, i.e., country-by-country as opposed to regional-level analysis [13]. In Ghana, social class was not associated with risk of malaria infection [14]. In Tanzania, there was no association between self-reported malaria and socioeconomic status (SES) but malaria prevalence was significantly higher among the lower SES individuals [15]. In Nigeria, self-reported malaria or fever was more frequent among the better-off SES and urban dwellers [16] though an earlier study showed a heavier malaria burden among the poor (<US $\$ 1 /$ day) compared to the rich [17]. Contrasting results are function of the methodology used to measure malaria and poverty. Studies based on self-reported fever are likely to overestimate the malaria burden while differential reporting of morbidity across socio-economic groups may also influence the results $[7,12]$.

In 2010/11, The Gambia conducted its first nationwide baseline Malaria Indicator Survey (MIS) in six health regions [18]. The survey covered 4,500 households. Data were collected on long-lasting insecticidal nets (LLINs) coverage and use, malaria case management, intermittent preventive treatment in pregnancy (IPTp), indoor residual spraying (IRS), household assets, general knowledge and practice on malaria prevention and control. In addition, a blood sample to determine the infection status and the haemoglobin level was collected among selected individuals.

In this study, laboratory-confirmed malaria infection and wealth quintiles were used to assess the association between SES including housing quality and malaria infection. The results are reported below.

\section{Methods}

\section{Management of the MIS 2010/11}

The Gambia National Malaria Control Programme (NMCP) in collaboration with The Gambia Bureau of Statistics (GBoS) conducted the MIS 2010/11. A technical working group (TWG), which included representatives from the NMCP, GBoS and other governmental and nongovernmental organizations, e.g. National Public Health Laboratories (NPHL), the National Pharmaceutical Services (NPS), the Medical Research Council (MRC), the Catholic
Relief Services (CRS), the United Nations Children's Fund (UNICEF) and the WHO, prepared the protocol and related documents. The GBoS analysed the data.

\section{Sample design}

The sampling frame used for the MIS 2010/11 comprised a complete list of all enumeration areas (EAs) categorized by the eight Local Government Areas (LGAs) and urban-rural residence from the 2003 Population and Housing Census. An EA or cluster is a geographic area with on average 400-700 individuals. According to the 2003 census, there were 2, 475 EAs. For the purpose of sampling, one of the eight LGAs was split into North Bank West Region (NBWR) and North Bank East Region (NBER) to facilitate the recoding of the LGAs into the six health regions at the analysis stage. Thus, for the sample selection, a total of nine LGAs were used.

A two-stage sampling was used to select 4,500 households. The first stage was carried out as a probability proportional to size systematic sampling using the 2003 Population Census Sampling Frame; 225 EAs, 97 in urban and 128 in rural areas were selected. In the second stage, households within the selected EAs were selected randomly from the list of all households within each selected EA prepared by the enumerators. From each EA, twenty households were selected for the interviews. The field coordinators verified the selected households to ensure that there is no selection bias. The enumerators made up to three visits to ensure completeness of the questionnaires, particularly for blood sampling.

The sample size was calculated based on the proportion of children under-five, $49 \%$ who slept under an ITN. Each LGA was first allocated with 430 households. This gives 3,870 households. The remaining 630 households were allocated proportional to the 2003 census households to achieve the sample total household count of 4,500 . Other parameters considered for the sample size calculation were the average number of children under-five per household (1.1) [19], average household size (8.3 persons) [20], a design effect of 2.0, relative standard error of $10 \%$ and a $90 \%$ response rate [21].

\section{Data collection}

The MIS 2010/11 started in November 2010 and ended in early January 2011. Two types of questionnaires were used, the household and the women's questionnaires, administered by 18 teams (two teams per nine LGA; one each for the household data collection and the health component i.e. haemoglobin and parasitaemia testing); each team comprised two supervisors, two enumerators, a nurse, a laboratory assistant and a driver. In addition, there were nine field coordinators whose roles were to ensure adherence to the survey protocol and sampling procedures. Overall, 29 enumerators collected the household 
data and nine nurses conducted the finger pricks for anaemia and malaria parasitaemia testing.

A blood sample for determining malaria infection and $\mathrm{Hb}$ level was collected on all children aged six months to 14 years and women 15-49 years old, including pregnant women, in all selected households. In addition, for every $4^{\text {th }}$ household (general population), all individuals, regardless of age, had a blood sample collected.

A rapid diagnostic test (RDT) was performed to determine malaria infection (SD Bioline Malaria pf/pan), and by thick and thin blood slides. The latter were stained with diluted Giemsa (10\%) for 10 minutes. Slides were read independently by two microscopists; slides with discrepant results between microscopy and RDT were read by a third microscopist [18]. Individuals with a positive RDT and with no history of anti-malarial treatment in the previous 14 days were treated with artemetherlumefantrine $(\mathrm{AL})$, the first line treatment in The Gambia. Pregnant women with a positive RDT and with no history of anti-malarial treatment in the previous 14 days received oral quinine if in the first trimester and AL in the second and third trimesters.

Haemoglobin $(\mathrm{Hb})$ was measured with the HaemoCue 201 machine (Ängelholm, Sweden) following the manufacturer's instructions [18]. Anaemia was defined as haemoglobin $(\mathrm{Hb})$ level $<11 \mathrm{~g} / \mathrm{dl}$ and severe anaemia as $\mathrm{Hb}<8 \mathrm{~g} / \mathrm{dl}$. All subjects with $\mathrm{Hb}<11 \mathrm{~g} / \mathrm{dl}$ were treated with ferrous sulphate tablets. Individuals with $\mathrm{Hb} \leq 8 \mathrm{~g} / \mathrm{dl}$ were treated with iron tablets and referred to the nearest health facility for further management.

Informed consent to participate in the survey was obtained from parents and/or guardians of children aged six months to 12 years, whilst assent was obtained from children aged 13-14 years. For adults, the information sheet about the survey on the questionnaire was read and translated into the local languages to seek their consent to participate in the survey [18].

\section{Study areas}

This study focuses on settlements/villages in the NBWR, CRR and URR thus: "North Bank West Region (NBWR)", "Central River Region (CRR)" and "Upper River Region (URR)", the poorest areas of The Gambia where malaria transmission is highest.

(i) The NBWR lies in the northwest of the River Gambia. It is the smallest health region with a total population of 110,970 persons and an annual growth rate of $2.3 \%$ between the 2003 and 2013 Censuses. The NBWR has a total land area of 1,054 sq $\mathrm{km}$ and a population density of 105 persons per sq $\mathrm{km}$ in 2013 [22]. It is the second poorest area with $60 \%$ of the population living on less than $\$ 1.25$ a day [23]. About $15.8 \%$ of the population were children under-five, $31 \%$ aged five to 14 years and $53.1 \%$ aged 15 years and above [22]. (ii) The CRR covers both the north and south banks of the River Gambia. It has a total population of 226,018 persons in 2013, an annual growth rate of $1.9 \%$, and a total land area of 2,894.25 sq km and a population density of 78 persons per sq $\mathrm{km}$. The CRR is home to $12.0 \%$ of the overall population of The Gambia [22]. It is the poorest area of the country. The 2010 poverty study showed that $79 \%$ and $73.2 \%$ of the population, respectively, for the CRR north and CRR south lived on less than $\$ 1.25$ a day [23]. In 2013, the CRR had $15.7 \%$ of its population under-five, $31 \%$ aged five to 14 years and $53.3 \%$ aged 15 years and above [22].

(iii) The URR lies in the eastern most part of The Gambia. Like the CRR, this region also covers both the north and south banks of the River Gambia. The URR has a total population of 239,916 persons, i.e., $12.7 \%$ of the overall population of The Gambia, with an annual growth rate of $2.8 \%$ in 2013. The total land area is $2,069.50 \mathrm{sq} \mathrm{km}$ and the population density in 2013 was 116 persons per sq $\mathrm{km}$ [22]. About $57 \%$ of the population live on less than $\$ 1.25$ a day [23]. Of the total population of the URR in 2013, $15.4 \%$ of its children were under-five, $31 \%$ aged five to 14 years, and $53.5 \%$ aged 15 years and above [22].

\section{Assets-based wealth quintiles}

Data on 27 variables of household possessions and assets, including housing conditions and materials for the construction of walls, roofs, floors and windows were collected. In an earlier analysis of the MIS data, the variables were coded and weights were calculated for use in the construction of indices. The weights were derived using the principal component analysis (PCA) based on Filmer and Pritchett's [24] method of classifying households into socio-economic groups (SEGs) or quintiles. The results are reassuring as they are consistent with those of the household economic survey [23] cited above, which used income and expenditure methods to measure poverty (Table 1 ).

\section{Production of malaria parasite prevalence maps}

Using the MIS 2010/11 dataset, a cross-tabulation of all the 225 selected EAs by the variable malaria result (malresult) by the eight local government areas (LGAs) was performed in stages to obtain malaria parasite prevalence by LGA and EA for the country. The results were transferred to an Excel database; and the district and name of settlements/village(s) were added as identifiers. The malaria parasite prevalence for the country was classified into five categories i.e. no malaria ( $0 \%)$, low ( $>0 \%$ and $<5 \%$ ), moderately-low $(\geq 5 \%$ and $<10 \%)$, moderately-high $(\geq 10 \%$ and $<20 \%)$, and high $(\geq 20 \%)$. The scale cut-offs of the malaria prevalence are standard ones as the different categories are $<5 \%$. This 
Table 1 Percentage distribution of households by wealth quintiles, residence and region, The Gambia MIS 2010/11 Wealth quintiles

\begin{tabular}{|c|c|c|c|c|c|c|c|}
\hline Residence & Poorest & Second & Middle & Fourth & Richest & Total & Number of households \\
\hline Urban & 0.4 & 4.5 & 16.2 & 35.1 & 43.8 & 100 & 1,908 \\
\hline Rural & 34.7 & 31.7 & 22.9 & 8.7 & 2.1 & 100 & 2,538 \\
\hline Total & 20 & 20 & 20 & 20 & 20 & 100 & 4,446 \\
\hline \multicolumn{8}{|l|}{ Health region } \\
\hline Western Region & 1.1 & 6.7 & 16.1 & 31.1 & 45.0 & 100 & 1,680 \\
\hline North Bank West Region & 24.3 & 32.4 & 25.7 & 13.6 & 4.0 & 100 & 448 \\
\hline North Bank East Region & 10.7 & 31.6 & 27.6 & 23.0 & 7.0 & 100 & 456 \\
\hline Lower River Region & 15.0 & 41.2 & 28.9 & 10.4 & 4.6 & 100 & 454 \\
\hline Central River Region & 54.2 & 23.6 & 13.2 & 6.3 & 2.7 & 100 & 937 \\
\hline Upper River Region & 29.1 & 17.0 & 25.9 & 20.2 & 7.9 & 100 & 471 \\
\hline Total & 20 & 20 & 20 & 20 & 20 & 100 & 4,446 \\
\hline
\end{tabular}

Source: The Gambia MIS 2010/11 Report, June 2012.

could be taken as the threshold for pre-elimination status, 5-9.99, 10-19.9 and then $>=20 \%$.

These classifications were used to produce a map of The Gambia showing malaria parasite prevalence by six health regions and settlements/villages (Figure 1). In order to have a good view of the settlements/villages with the highest malaria prevalence, three maps were produced separately for the NBWR, CRR and URR with settlement/village names added (Figure 2A, B and $\mathrm{C}$ respectively). The unit of analysis for the map is EA or cluster. ArcGIS 10.2.2 Desktop software was used to produce the maps.

\section{Data management and analysis}

A subset of the MIS 2010 dataset (i.e., the low, moderately low and moderately high and high malaria prevalence settlements/villages) was selected for this project. The selected dataset contains 12,274 records. SPSS (version 19) was used for the initial analysis using Pearson's Chi-square test of significance. All the subsequent data analyses, e.g., production of tables, simple and multiple logistic regressions were performed using STATA version 12.1 (StataCorp LP, College Station, TX, USA). Survey data analysis procedures with Taylor [25] series expansion linearized standard errors to account for cluster sampling and unequal selection probabilities was used. However, the analyses in this report were not weighted. Percentages for categorical variables and means for continuous variables with 95\% confidence intervals were estimated.

Simple and multiple logistic regression analyses were applied to assess predictors of malaria prevalence among children six to 59 months, five to 14 years as well as all the age groups combined, including 15 year olds and above in the selected general population (one in four households). The association of SES by wealth quintiles estimated using wealth asset index was assessed. The association of malaria with the type of housing materials used for the construction of walls, floors, roofs, and windows was also assessed. Separate analyses were performed for the children six to 59 months, five to

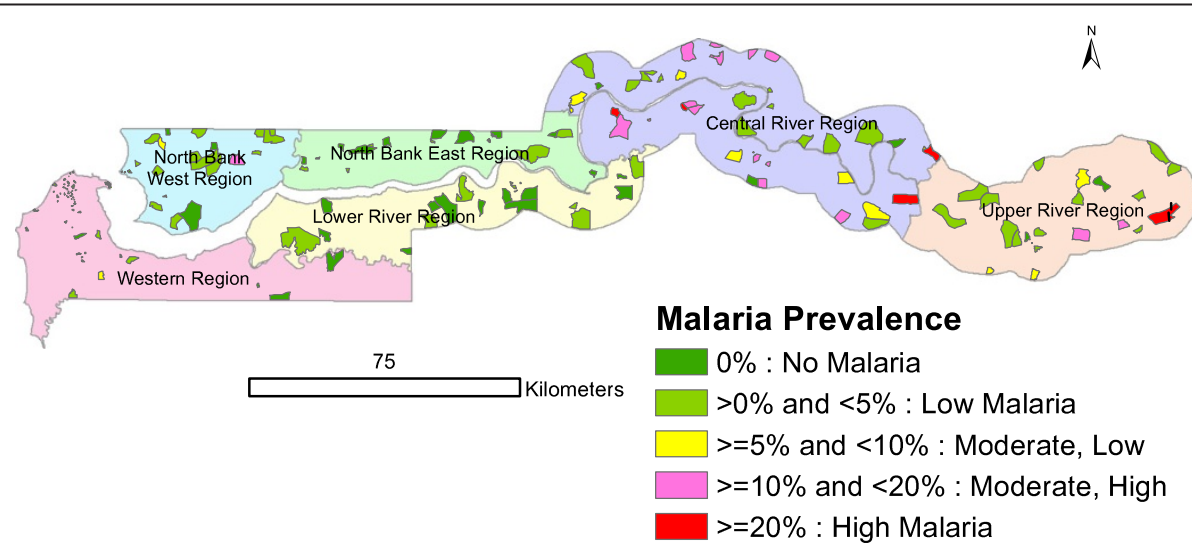

Figure 1 Malaria parasite prevalence by six health regions, The Gambia MIS 2010/11. 


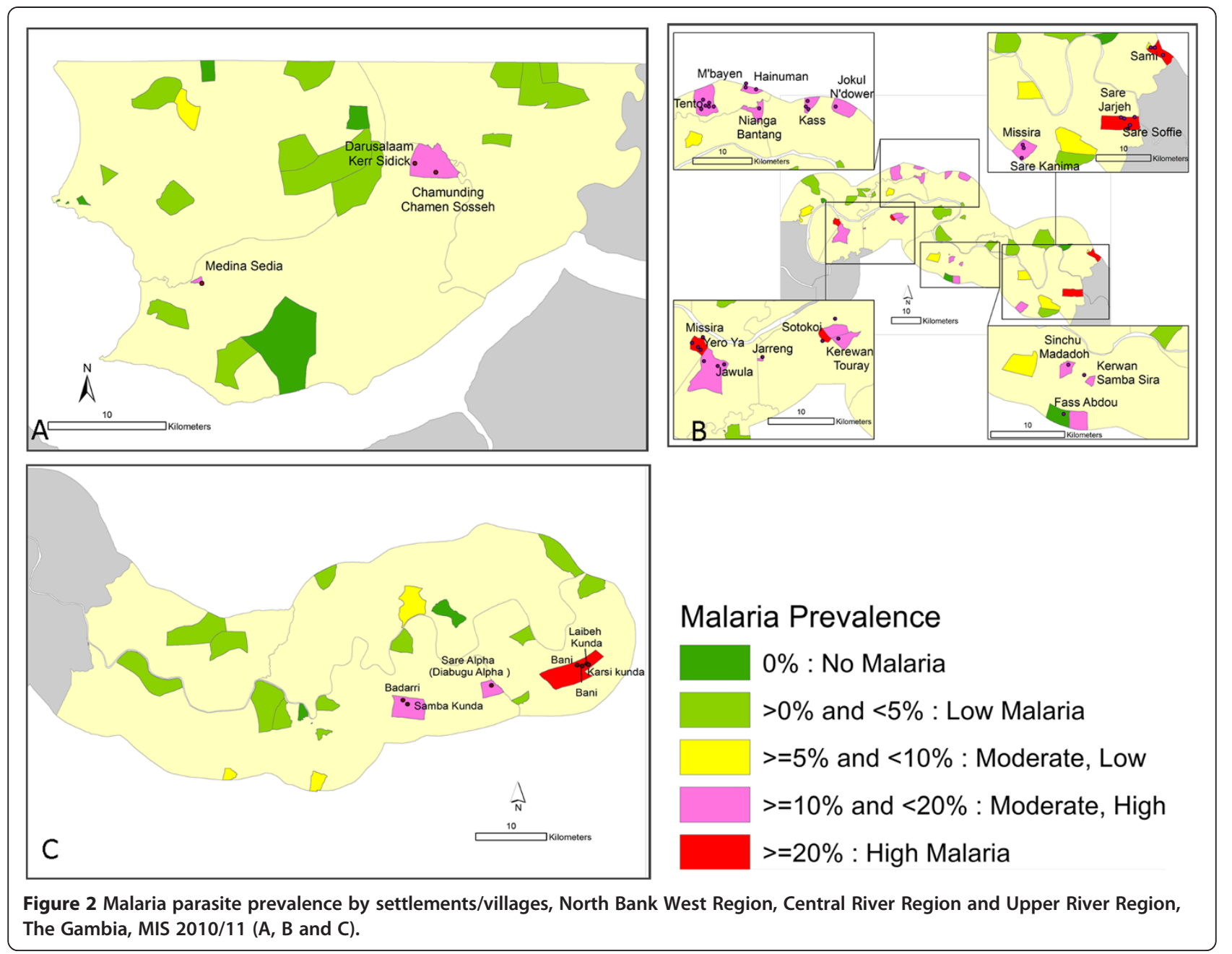

14 years and the combined age groups, including 15 year olds and above in the selected general population (one in four households). The rationale is to compare with the six to 59 months children and to ascertain if the burden of malaria has shifted from the under-fives to the older children (five to 14 years). The general population was also meant to provide comparable malaria parasite prevalence data with the children under-five (six to 59 months).

All adjusted analyses were controlled for age groups, sex and health regions. Unadjusted and adjusted odds ratios with 95\% confidence intervals are presented. Statistical significance was defined as a p-value $<0.05$. Housing quality was defined based on construction materials used for the floors, walls, roofs, and windows (Table 2).

\section{Ethics and approval}

The Gambia MIS 2010/11 survey protocol was subjected to rigorous review before approval was granted. The University of The Gambia (UTG) Committee on Research
Table 2 Definitions of housing quality used in the logistic regression model, The Gambia MIS 2010/11

\begin{tabular}{|c|c|}
\hline $\begin{array}{l}\text { Building } \\
\text { characteristics }\end{array}$ & Materials used for construction \\
\hline \multirow[t]{2}{*}{ Floor } & Good $=$ (Cement or tiles) \\
\hline & Poor $=($ Earth/sand, dung and palm bamboo $)$ \\
\hline \multirow[t]{2}{*}{ Wall } & Good $=($ Bricks, cement blocks, covered adobe and other $)$ \\
\hline & $\begin{array}{l}\text { Poor }=(\text { Cane/palm/trunks, mud/dirt, stone with mud; } \\
\text { and mud/krinting*) }\end{array}$ \\
\hline \multirow[t]{2}{*}{ Roof } & Good = (Sod, metal corrugated iron, cement concrete) \\
\hline & Poor = (Thatch/palm leaf $)$ \\
\hline \multirow[t]{2}{*}{ Window } & $\begin{array}{l}\text { Good = (having a window with at least glasses, screen, } \\
\text { curtain, or shutters) }\end{array}$ \\
\hline & $\begin{array}{l}\text { Poor }=\text { (No window at all or window without glasses, } \\
\text { screen, curtain, or shutters) }\end{array}$ \\
\hline
\end{tabular}

*Krinting is a Wolof word for woven bamboo. Walls are constructed with woven krinting and then plastered with mud in the interior and exterior. 
reviewed the first draft of the protocol. The Scientific Coordinating Committee (SCC) of the MRC Unit, The Gambia, reviewed the final protocol and the Joint Gambia Government/MRC Ethics Committee granted approval to conduct the MIS 2010/11.

\section{Results}

Over 18,000 blood slides were collected. The overall malaria prevalence among children aged six to 59 months as determined by microscopy was $4 \%(5,118)$. SD Bioline Malaria p/f pan had a sensitivity of $99.0 \%$ (95\% CIs: 97.5-99.7) and specificity 98.1\% (95\% CIs: 96.7-99.0), consistent with the manufacturer's estimates [18].

Malaria parasite prevalence among children was highest in the Central River Region (CRR), 9.9\% $(1,445)$ and the Upper River Region (URR), 4.4\% (964); and lowest in the North Bank East Region (NBER), 0.5\% (527) and the Lower River Region (LRR), 0.8\% (481). The Western Region (WR) and the North Bank West Region (NBWR) had malaria parasite prevalence of $2.5 \%(1,027)$; and $3.1 \%$ (674), respectively [18].

The CRR had the highest percentage of poorest households (54.2\%), followed by the URR (29.1\%) and the NBWR (24.3\%) (Table 1). Prevalence was heterogeneous, with values of $0 \%$ or $<5 \%$ in the WR (Figure 1) although some EAs in the NBWR had higher prevalence (Figure 2A). Most EAs with moderate-high to high prevalence were found in the CRR and the URR i.e. the central and eastern parts of the country (Figure 2B-C).

\section{Malaria parasite prevalence among children aged six to 59 months and children aged five to 14 years}

The overall malaria parasite prevalence among children aged six to 59 months was $10 \%(1,248)$, (95\% CIs: 7.812.7\%). Malaria parasite prevalence tended to be higher among children aged six to 11 months: $13.2 \%$ (144), (95\% CIs: 7.6-21.9\%) and among children aged thirty-six to 47 months: $11.9 \%$ (301) (95\% CIs: 7.9-17.8\%). Children from the poorest households were 8.2 times (15.6 vs 1.9\%) more likely to have had malaria compared to children from the richest households (Table 3).

The overall malaria parasite prevalence among children aged five to 14 years was $11 \%(1,987),(95 \%$ CIs: 8.4-14.3\%). Children from the poorest households were 2.6 times (15.2 vs 5.8\%) more likely to have had malaria compared to children from the richest households (Table 4).

\section{Malaria parasite prevalence in the general population} In the general population, the overall malaria parasite prevalence was $8.7 \%$ (2,306), (95\% CIs: 7.4-10.2\%). Parasite prevalence was highest among children aged five to 14 years, $10.5 \%$ (693), (95\% CIs: 8.6-12.9\%). Children from the poorest households were 3.7 times (11.1 vs 3.0\%) more
Table 3 Malaria parasite prevalence among children aged six to 59 months by background characteristics, The Gambia MIS 2010/11

\begin{tabular}{|c|c|c|c|}
\hline Background characteristics & $\mathrm{N}$ & $n$ & $\%(95 \% \mathrm{Cl})$ \\
\hline \multicolumn{4}{|l|}{ Age (months) } \\
\hline $6-11$ & 144 & 19 & $13.1(7.5,21.9)$ \\
\hline $12-23$ & 273 & 24 & $8.7(5.7,13.2)$ \\
\hline $24-35$ & 284 & 27 & $9.5(6.0,14.5)$ \\
\hline $36-47$ & 301 & 36 & $11.9(7.8,17.7)$ \\
\hline $48-59$ & 246 & 19 & $7.7(4.9,11.9)$ \\
\hline Total & 1,248 & 125 & $10.0(7.8,12.7)$ \\
\hline \multicolumn{4}{|l|}{ Sex } \\
\hline Male & 681 & 69 & $10.1(7.6,13.3)$ \\
\hline Female & 567 & 56 & $9.8(7.0,13.6)$ \\
\hline Total & 1,248 & 125 & $10.0(7.8,12.7)$ \\
\hline \multicolumn{4}{|l|}{ Health regions } \\
\hline Western Region & 56 & 5 & $8.9(3.7,19.8)$ \\
\hline North Bank West Region & 104 & 8 & $7.6(2.2,22.9)$ \\
\hline Central River Region & 714 & 92 & $12.8(9.8,16.7)$ \\
\hline Upper River Region & 359 & 19 & $5.2(2.4,10.9)$ \\
\hline Total & $1,233^{*}$ & 124 & $10.0(7.8,12.8)$ \\
\hline \multicolumn{4}{|l|}{ Wealth Index } \\
\hline Poorest & 500 & 78 & $15.6(12.3,19.4)$ \\
\hline Second & 224 & 16 & $7.1(4.0,12.3)$ \\
\hline Middle & 260 & 22 & $8.4(4.9,14.1)$ \\
\hline Fourth & 146 & 6 & $4.1(2.0,8.2)$ \\
\hline Richest & 103 & 2 & $1.9(0.7,5.3)$ \\
\hline Total & 1,248 & 125 & $10.0(7.8,12.7)$ \\
\hline
\end{tabular}

*Total excludes missing cases.

likely to have had malaria compared to children from the richest households (Table 5).

\section{Multivariate analysis}

Among children six to 59 months old, there were no significant associations between malaria and age $(p=0.438)$, health region $(\mathrm{p}=0.133)$ and sex $(\mathrm{p}=0.621)$. After adjusting for age, sex and health region, children from the second $(\mathrm{OR}=0.42 ; 95 \% \mathrm{CI}: 0.25 ; 0.73, \mathrm{p}=0.002)$, third $(\mathrm{OR}=0.52 ; 95 \% \mathrm{CI}: 0.29 ; 0.95, \mathrm{p}=0.03)$, fourth $(\mathrm{OR}=$ 0.27; $95 \%$ CI: $0.12 ; 0.61, \mathrm{p}=0.002)$ and richest $(\mathrm{OR}=$ 0.12; $95 \%$ CI: $0.05 ; 0.33, \mathrm{p}<0.001)$ quintiles were significantly less likely to have malaria compared to children from the poorest quintiles. Having poor walls $(\mathrm{OR}=$ 1.87; 95\% CI: 1.12; 3.11, $\mathrm{p}=0.01)$, floors $(\mathrm{OR}=2.0 ; 95 \%$ CI: $1.4 ; 3.0, \mathrm{p}=0.001)$, roofs $(\mathrm{OR}=2.2 ; 95 \% \mathrm{CI}: 1.4 ; 3.3$, $\mathrm{p}<0.001)$, and windows ( $\mathrm{OR}=1.9 ; 95 \% \mathrm{CI}: 1.2 ; 3.0, \mathrm{p}=0.01)$ was significantly associated with higher prevalence of malaria even after adjusting for age, sex and health regions (Table 6). 
Table 4 Malaria parasite prevalence among children aged five to $\mathbf{1 4}$ years by background characteristics, The Gambia MIS 2010/11

\begin{tabular}{lccc}
\hline Background characteristics & $\mathbf{N}$ & $\mathbf{n}$ & $\mathbf{\%}(\mathbf{9 5 \%} \mathbf{C l})$ \\
\hline Age (years) & & & \\
$5-9$ & 1,094 & 115 & $10.5(7.5,14.5)$ \\
$10-14$ & 893 & 104 & $11.6(8.7,15.3)$ \\
Total & $\mathbf{1 , 9 8 7}$ & $\mathbf{2 1 9}$ & $11.0(8.4,14.3)$ \\
Sex & & & \\
Male & 1,003 & 117 & $11.6(8.6,15.5)$ \\
Female & 984 & 102 & $10.3(7.6,13.8)$ \\
Total & $\mathbf{1 , 9 8 7}$ & $\mathbf{2 1 9}$ & $11.0(8.4,14.3)$ \\
Health regions & & & \\
Western Region & 74 & 6 & $8.1(6.1,10.6)$ \\
North Bank West Region & 175 & 15 & $8.5(4.6,15.2)$ \\
Central River Region & 1,054 & 131 & $12.4(9.3,16.2)$ \\
Upper River Region & 656 & 65 & $9.9(4.9,18.9)$ \\
Total & $\mathbf{1 , 9 5 9 *}$ & $\mathbf{2 1 7}$ & $11.0(8.4,14.4)$ \\
Wealth index & & & \\
Poorest & 717 & 109 & $15.2(11.5,19.7)$ \\
Second & 358 & 34 & $9.5(5.1,16.8)$ \\
Middle & 416 & 50 & $12.0(8.3,16.9)$ \\
Fourth & 314 & 15 & $4.7(2.4,9.0)$ \\
Richest & 154 & 9 & $5.8(3.7,8.9)$ \\
Total & $\mathbf{1 , 9 8 7}$ & $\mathbf{2 1 9}$ & $\mathbf{1 1 . 0}(\mathbf{8 . 4}, \mathbf{1 4 . 3})$ \\
\hline *Total excludes missing cases. & & &
\end{tabular}

Among children aged five to 14 years old, there were no significant associations between malaria and age $(\mathrm{p}=0.4)$, health region $(p=0.2)$ and sex $(p=0.4)$. Children from the fourth $(\mathrm{OR}=0.2 ; 95 \% \mathrm{CI}: 0.1 ; 0.5, \mathrm{p}<0.001)$ and richest $(\mathrm{OR}=0.3 ; 95 \% \mathrm{CI}: 0.1 ; 0.6, \mathrm{p}=0.003)$ quintiles were significantly less likely be infected compared to those from the poorest quintiles. However, there were no significant differences in malaria prevalence between children from the poorest quintiles and those from the second $(\mathrm{p}=0.1)$ and third ( $\mathrm{p}=0.1$ ) quintiles. Having poor walls $(\mathrm{OR}=1.8$; 95\% CI: $1.1 ; 3.0, \mathrm{p}=0.01)$, floors $(\mathrm{OR}=1.5 ; 95 \% \mathrm{CI}: 1.0$; 2.2, $\mathrm{p}=0.03)$, roofs $(\mathrm{OR}=1.6 ; 95 \% \mathrm{CI}: 1.1 ; 2.3, \mathrm{p}=0.01)$, and windows $(\mathrm{OR}=1.7 ; 95 \% \mathrm{CI}: 1.3 ; 2.3, \mathrm{p}<0.001)$ were significantly associated with higher prevalence of malaria, even after adjusting for age, sex and health regions (Table 7).

In the general population, i.e. all household members in every $4^{\text {th }}$ household, malaria prevalence was significantly lower in the fourth $(\mathrm{OR}=0.4 ; 95 \% \mathrm{CI}$ : $0.2 ; 0.8$, $\mathrm{p}=0.01)$ and richest $(\mathrm{OR}=0.2 ; 95 \% \mathrm{CI}: 0.08 ; 0.8, \mathrm{p}=0.02)$ quintiles compared to the poorest quintiles. Having poor wall housing materials was associated with higher prevalence of malaria $(\mathrm{OR}=1.6 ; 95 \% \mathrm{CI}: 1.1 ; 2.3, \mathrm{p}=0.01)$.
Table 5 Malaria parasite prevalence in the general population by age and background characteristics, The Gambia MIS 2010/11

\begin{tabular}{|c|c|c|c|}
\hline Background characteristics & $\mathrm{N}$ & $n$ & $\%(95 \% \mathrm{Cl})$ \\
\hline \multicolumn{4}{|l|}{ Age } \\
\hline 6-59 months & 394 & 32 & $8.1(5.6,11.4)$ \\
\hline $5-14$ years & 693 & 73 & $10.5(8.5,12.9)$ \\
\hline $15+$ & 1,219 & 96 & $7.8(6.2,9.9)$ \\
\hline Total & 2,306 & 201 & $8.7(7.4,10.2)$ \\
\hline \multicolumn{4}{|l|}{ Sex } \\
\hline Male & 1,065 & 108 & $10.1(8.2,12.4)$ \\
\hline Female & 1,241 & 93 & $7.4(5.9,9.4)$ \\
\hline Total & 2,306 & 201 & $8.7(7.4,10.2)$ \\
\hline \multicolumn{4}{|l|}{ Health regions } \\
\hline Western Region & 114 & 8 & $7.0(3.5,13.4)$ \\
\hline North Bank West Region & 195 & 9 & $4.6(1.9,10.7)$ \\
\hline Central River Region & 1,180 & 128 & $10.8(8.8,13.2)$ \\
\hline Upper River Region & 765 & 48 & $6.2(4.6,8.3)$ \\
\hline Total & $2,254^{*}$ & 193 & $8.5(7.2,10.1)$ \\
\hline \multicolumn{4}{|l|}{ Wealth index } \\
\hline Poorest & 863 & 96 & $11.1(8.7,14.1)$ \\
\hline Second & 484 & 38 & $7.8(5.2,11.5)$ \\
\hline Middle & 456 & 40 & $8.7(6.5,11.7)$ \\
\hline Fourth & 286 & 14 & $4.9(3.2,7.3)$ \\
\hline Richest & 165 & 5 & $3.0(0.9,9.1)$ \\
\hline Total & 2,306 & 201 & $8.7(7.4,10.2)$ \\
\hline
\end{tabular}

However, floors, roofs and windows were not significantly associated with malaria infection (Table 8).

\section{Discussion}

Malaria prevalence was strongly associated with SES, even after adjusting for age, sex and health region, regardless of the age group. These findings are consistent with previous studies carried out in sub-Saharan Africa in which other measures of SES such as occupation, housing type, including the uptake of interventions, e.g., ITNs and expenditure on chemoprophylaxis were associated with malaria infection [12,26-31].

The recent findings by Tusting et al. [9], is also consistent with this study. However, these findings differ from Somi et al. [15], who found no association between malaria and SES, and Somi et al. [32], who found negative associations between malaria and SES running in both directions. In addition, the findings of this study are contrary to a study [16] in southeast Nigeria based on self-reported malaria or fever, which found more malaria among better-off SES and urban dwellers compared to the poor. 
Table 6 Factors associated with malaria parasite prevalence among children aged six to 59 months, The Gambia MIS, 2010/11

\begin{tabular}{|c|c|c|c|c|c|}
\hline \multirow[t]{2}{*}{ Variables } & \multirow[b]{2}{*}{$\mathrm{N}$} & \multicolumn{2}{|c|}{ Unadjusted } & \multicolumn{2}{|c|}{ Adjusted } \\
\hline & & OR $(95 \% \mathrm{Cl})$ & $P$ value & OR $(95 \% \mathrm{Cl})$ & $P$ value \\
\hline Age group (months) & & $1.0(0.9 ; 1.050)$ & 0.6 & & \\
\hline $06-11$ & 144 & 1 & & 1 & 0.4 \\
\hline $12-23$ & 273 & $0.6(0.3 ; 1.2)$ & 0.1 & $0.7(0.3 ; 1.2)$ & 0.2 \\
\hline $24-35$ & 284 & $0.7(0.3 ; 1.6)$ & 0.3 & $0.7(0.3 ; 1.7)$ & 0.5 \\
\hline $36-47$ & 301 & $0.9(0.4 ; 1.9)$ & 0.7 & $1.0(0.5 ; 2.2)$ & 0.9 \\
\hline $48-59$ & 246 & $0.6(0.2 ; 1.3)$ & 0.1 & $0.6(0.2 ; 1.4)$ & 0.2 \\
\hline \multicolumn{6}{|l|}{ Health regions } \\
\hline Western Region & 56 & 1 & & 1 & 0.1 \\
\hline North Bank West Region & 104 & $0.8(0.2 ; 4.1)$ & 0.8 & $0.5(0.1 ; 2.4)$ & 0.4 \\
\hline Central River Region & 714 & $1.5(0.6 ; 4.0)$ & 0.4 & $0.6(0.3 ; 1.4)$ & 0.3 \\
\hline Upper River Region & 359 & $0.6(0.2 ; 1.9)$ & 0.3 & $0.4(0.1 ; 1.0)$ & 0.06 \\
\hline \multicolumn{6}{|l|}{ Sex } \\
\hline Male & 681 & 1 & & 1 & 0.6 \\
\hline Female & 567 & $0.9(0.6 ; 1.4)$ & 0.8 & $0.9(0.6 ; 1.3)$ & \\
\hline \multicolumn{6}{|l|}{ Wealth quintiles } \\
\hline Poorest & 500 & 1 & & 1 & \\
\hline Second & 224 & $0.4(0.2 ; 0.8)$ & 0.009 & $0.4(0.2 ; 0.7)$ & 0.002 \\
\hline Third & 260 & $0.5(0.2 ; 0.9)$ & 0.03 & $0.5(0.3 ; 0.9)$ & 0.03 \\
\hline Fourth & 146 & $0.2(0.1 ; 0.5)$ & 0.001 & $0.3(0.1 ; 0.6)$ & 0.002 \\
\hline Richest & 103 & $0.1(0.04 ; 0.3)$ & $<0.001$ & $0.1(0.04 ; 0.3)$ & $<0.001$ \\
\hline \multicolumn{6}{|l|}{ Wall type } \\
\hline Good & 451 & 1 & & 1 & \\
\hline Poor & 797 & $2.3(1.3 ; 4.1)$ & 0.005 & $1.8(1.1 ; 3.1)$ & 0.01 \\
\hline \multicolumn{6}{|l|}{ Floor type } \\
\hline Good & 644 & 1 & & 1 & \\
\hline Poor & 604 & $2.5(1.5 ; 3.9)$ & $<0.001$ & $2.0(1.4 ; 3.0)$ & 0.001 \\
\hline \multicolumn{6}{|l|}{ Roof type } \\
\hline Good & 799 & 1 & & 1 & \\
\hline Poor & 449 & $2.4(1.6 ; 3.7)$ & $<0.001$ & $2.2(1.4 ; 3.3)$ & $<0.001$ \\
\hline \multicolumn{6}{|l|}{ Window type } \\
\hline Good & 730 & 1 & & 1 & \\
\hline Poor & 513 & $2.0(1.3 ; 3.0)$ & 0.001 & $1.8(1.1 ; 3.0)$ & 0.01 \\
\hline
\end{tabular}

Age, health region and sex were not significantly associated with malaria prevalence across sub-population groups. However, malaria prevalence was significantly higher in the CRR compared to the URR, with males being more at risk than females. It is interesting noticing that the CRR is the poorest in The Gambia followed by the URR [23,33].

The high malaria infection in the CRR and the URR (i.e. eastern parts of the country) can be explained by ecological factors such as fresh water, floodplains, swamp and upland rice fields, higher temperatures and lower humidity compared to the WR (western half of the country). Studies have shown that swamp rice fields are important habitat for mosquito larvae [34,35].

The malaria burden has also shifted from under-five children (six to 59 months) to children aged five to 14 years; $\mathrm{OR}=1.4$ (95\% CI: $0.9 ; 2.1), \mathrm{p}=0.168$, see Table 8 ). The reasons for the shift in malaria burden from the under-fives to the older children aged five to 14 years are unclear. One hypothesis is that The Gambia NMCP had 
Table 7 Factors associated with malaria parasite prevalence among children aged five to 14 years, The Gambia MIS, 2010/11

\begin{tabular}{|c|c|c|c|c|c|}
\hline \multirow[t]{2}{*}{ Variables } & \multirow[b]{2}{*}{$\mathrm{N}$} & \multicolumn{2}{|c|}{ Unadjusted } & \multicolumn{2}{|c|}{ Adjusted } \\
\hline & & OR $(95 \% \mathrm{Cl})$ & $P$ value & OR $(95 \% \mathrm{Cl})$ & $P$ value \\
\hline Age (years) & & $1.0(0.9 ; 1.0)$ & 0.8 & & \\
\hline \multicolumn{6}{|l|}{ Age group (years) } \\
\hline $5-9$ & 1094 & 1 & & 1 & 0.4 \\
\hline $10-14$ & 893 & $1.1(0.8 ; 1.6)$ & 0.5 & $1.1(0.8 ; 1.6)$ & \\
\hline \multicolumn{6}{|l|}{ Health regions } \\
\hline Western Region & 74 & 1 & & 1 & 0.2 \\
\hline North Bank West Region & 175 & $1.0(0.5 ; 2.2)$ & 0.9 & $0.6(0.3 ; 1.3)$ & 0.2 \\
\hline Central River Region & 1054 & $1.6(1.0 ; 2.5)$ & 0.03 & $0.8(0.4 ; 1.4)$ & 0.4 \\
\hline Upper River Region & 656 & $1.2(0.5 ; 2.8)$ & 0.6 & $1.0(0.5 ; 1.9)$ & 0.9 \\
\hline \multicolumn{6}{|l|}{ Sex } \\
\hline Male & 1003 & 1 & & 1 & 0.4 \\
\hline Female & 984 & $0.9(0.6 ; 1.2)$ & 0.4 & $0.9(0.6 ; 1.2)$ & \\
\hline \multicolumn{6}{|l|}{ Wealth quintiles } \\
\hline Poorest & 717 & 1 & & 1 & \\
\hline Second & 358 & $0.6(0.3 ; 1.1)$ & 0.1 & $0.6(0.3 ; 1.2)$ & 0.1 \\
\hline Third & 416 & $0.8(0.5 ; 1.2)$ & 0.2 & $0.7(0.5 ; 1.1)$ & 0.1 \\
\hline Fourth & 314 & $0.3(0.1 ; 0.6)$ & 0.001 & $0.2(0.1 ; 0.5)$ & 0.000 \\
\hline Richest & 154 & $0.3(0.2 ; 0.6)$ & $<0.001$ & $0.3(0.1 ; 0.6)$ & 0.003 \\
\hline \multicolumn{6}{|l|}{ Wall type } \\
\hline Good & 796 & 1 & & 1 & \\
\hline Poor & 1191 & $1.7(1.1 ; 2.7)$ & 0.011 & $1.8(1.1 ; 3.0)$ & 0.02 \\
\hline \multicolumn{6}{|l|}{ Floor type } \\
\hline Good & 1094 & 1 & & 1 & \\
\hline Poor & 893 & $1.6(0.9 ; 2.5)$ & 0.07 & $1.5(1.0 ; 2.2)$ & 0.03 \\
\hline \multicolumn{6}{|l|}{ Roof type } \\
\hline Good & 1343 & 1 & & 1 & \\
\hline Poor & 643 & $1.7(1.2 ; 2.5)$ & 0.006 & $1.6(1.1 ; 2.3)$ & 0.015 \\
\hline \multicolumn{6}{|l|}{ Window type } \\
\hline Good & 1228 & 1 & & 1 & \\
\hline Poor & 750 & $1.8(1.4 ; 2.3)$ & $<0.001$ & $1.7(1.3 ; 2.3)$ & $<0.001$ \\
\hline
\end{tabular}

initially over focused (i.e., activities and information, education and communication (IEC) messages) on the underfives (0-59 months), particularly for ITN coverage. However, the finding is consistent with a study in northeast Tanzania, which showed that children aged five to 13 years were now at a higher risk of malaria compared to underfive children. The odds of having malaria were also higher for males compared to females in northeast Tanzania and Ethiopia $[28,36]$.

Historically, malaria elimination in the USA, Italy, Greece, and Spain was achieved through socio-economic development and intensive anti-malarial interventions, such as improved housing. Doors and windows were screened to reduce contacts between mosquitoes and people coupled with combined environmental management, e.g., draining of swampland to eliminate breeding grounds of certain vector mosquitoes and IRS using DDT [6,9]. For example, in The Gambia, a randomized controlled trial of full screening of doors and windows and closing of eaves showed a 50\% decline in the risk of anaemia-related malaria among children [37]. This randomized controlled trial has proved that improvements in housing quality can greatly reduce malaria transmission and incidence.

Interventions aiming at improving the living conditions of populations in endemic communities can have a major impact on the malaria burden [4]. This is also supported 
Table 8 Factors associated with malaria parasite prevalence among the general population, The Gambia MIS, 2010/11

\begin{tabular}{|c|c|c|c|c|c|}
\hline \multirow[t]{2}{*}{ Variables } & \multirow[b]{2}{*}{$\mathbf{N}$} & \multicolumn{2}{|c|}{ Unadjusted } & \multicolumn{2}{|c|}{ Adjusted } \\
\hline & & OR $(95 \% \mathrm{Cl})$ & $P$ value & OR $(95 \% \mathrm{Cl})$ & $P$ value \\
\hline Age (years) & & $1.0(0.1 ; 1.0)$ & 0.1 & & \\
\hline \multicolumn{6}{|l|}{ Age group (years) } \\
\hline$<5$ & 394 & 1 & & 1 & \\
\hline $5-14$ & 693 & $1.3(0.8 ; 2.1)$ & 0.2 & $1.4(0.9 ; 2.1)$ & 0.1 \\
\hline $15+$ & 1219 & $1.0(0.6 ; 1.4)$ & 0.8 & $1.0(0.7 ; 1.4)$ & 0.8 \\
\hline \multicolumn{6}{|l|}{ Health Region } \\
\hline Western Region & 114 & $0.6(0.3 ; 1.3)$ & 0.2 & $1.4(0.6 ; 3.6)$ & 0.5 \\
\hline North Bank West Region & 195 & $0.4(0.2 ; 1.0)$ & 0.05 & $0.4(0.2 ; 1.0)$ & 0.05 \\
\hline Central River Region & 1180 & 1 & & 1 & 0.02 \\
\hline Upper River Region & 765 & $0.5(0.4 ; 0.8)$ & 0.003 & $0.74(0.5 ; 1.00)$ & 0.05 \\
\hline \multicolumn{6}{|l|}{ Sex } \\
\hline Male & 1065 & 1 & & 1 & 0.09 \\
\hline Female & 1241 & $0.7(0.5 ; 1.0)$ & 0.04 & $0.8(0.5 ; 1.0)$ & \\
\hline \multicolumn{6}{|l|}{ Wealth quintiles } \\
\hline Poorest & 863 & 1 & & 1 & 0.05 \\
\hline Second & 484 & $0.7(0.4 ; 1.1)$ & 0.2 & $0.8(0.4 ; 1.3)$ & 0.3 \\
\hline Third & 456 & $0.8(0.5 ; 1.2)$ & 0.2 & $0.8(0.5 ; 1.2)$ & 0.3 \\
\hline Fourth & 286 & $0.4(0.2 ; 0.7)$ & 0.001 & $0.4(0.2 ; 0.8)$ & 0.01 \\
\hline Richest & 165 & $0.2(0.08 ; 0.8)$ & 0.02 & $0.2(0.07 ; 0.8)$ & 0.02 \\
\hline \multicolumn{6}{|l|}{ Wall type } \\
\hline Good & 850 & 1 & & 1 & \\
\hline Poor & 1456 & $1.8(1.3 ; 2.6)$ & 0.002 & $1.6(1.1 ; 2.3)$ & 0.01 \\
\hline \multicolumn{6}{|l|}{ Floor type } \\
\hline Good & 1245 & 1 & & 1 & \\
\hline Poor & 1061 & $1.6(1.1 ; 2.4)$ & 0.01 & $1.3(0.8 ; 2.2)$ & 0.2 \\
\hline \multicolumn{6}{|l|}{ Roof type } \\
\hline Good & 1546 & 1 & & 1 & \\
\hline Poor & 757 & $1.8(1.2 ; 2.7)$ & 0.004 & $1.5(1.0 ; 2.4)$ & 0.07 \\
\hline \multicolumn{6}{|l|}{ Window type } \\
\hline Good & 1309 & 1 & & 1 & \\
\hline Poor & 991 & $1.3(0.9 ; 1.9)$ & 0.2 & $1.2(0.8 ; 1.7)$ & 0.4 \\
\hline
\end{tabular}

by the finding that housing construction materials, e.g., type of wall, floor, roof, and window, are strongly associated with the risk of infection in most age groups, though in the general population this was true only for poor wall housing materials. The association with type of housing and risk of malaria has already been described in other countries. In Mozambique [38], Eritrea [39,40] and the Lao People's Democratic Republic, living in houses with grass roof was associated with higher odds of malaria infection [41]. Similarly, houses constructed with bamboo walls, poorly fitted windows and doors, and often inhabited by people from low SES were associated with mosquito house entry [41]. Living in houses with no windows or screens is likely to increase individual contact with the mosquito vector [42]. Mud used as wall material was associated with high prevalence of malaria [43].

However, the study has several limitations. The analysis did not include women aged 15-49 years who were pregnant at the time of the survey due to the paucity of the data (only 148 women). Nonetheless, compared to the other subpopulations in this study, pregnant women had the lowest malaria parasite prevalence of $6.8 \%$ (95\% CIs: 2.7-10.9\%). Also, interventions such as ITN/LLIN ownership and use and IRS were not included due to the paucity of data.

Over the last decade, The Gambia has made significant progress in reducing the malaria burden. All-cause under- 
five mortality declined from 141 deaths per 1,000 in 2002 to 131 deaths per 1,000 in 2007 [19] and further declined significantly to 109 deaths per 1,000 in 2012 [44]. A new Malaria Strategic Plan and Policy for the period 2014-2020 [45,46] with a vision of a 'malaria-free Gambia by 2020' has been prepared. Nevertheless, malaria transmission is still ongoing, despite the achieved high coverage of preventive and curative interventions. There may be the need of additional interventions targeting the human reservoir of infection and aiming at interrupting transmission.

The findings of this study pose major challenges for malaria prevention and control interventions in The Gambia. First, there is the need to address the unacceptably high malaria burden among the poorest communities in the CRR, URR and NBWR. Studies have shown that malaria infections and mosquito vectors are not homogenously distributed within population groups and areas. Thus, it is argued that countries address the heterogeneity in malaria transmission long before they enter the pre-elimination phase $[43,47,48]$. Second, there is the need to consolidate the gains made as The Gambia moves towards the malaria pre-elimination phase. Increased funding is critical in achieving these objectives. Currently, the country is the only one in the sub-region where malaria interventions are funded from a single source, the Global Fund for AIDS, Tuberculosis and Malaria (GFATM). Funding is therefore a major challenge, which threatens the prospects for sustained malaria decline.

\section{Conclusions}

The evidence suggests that malaria infection was strongly associated with low SES. Children from the poorest quintiles, living in poor housing conditions were more at risk of having malaria infection. While intensifying the existing malaria interventions may reduce malaria in the short term, investments in strategies that address socio-economic disparities and improvements in the quality of housing could, in the long term, significantly reduce the malaria burden in the poorest communities.

\footnotetext{
Abbreviations

AL: Artemether-lumefantrine; CRR: Central River Region; EAs: Enumeration areas; GBoS: Gambia Bureau of Statistics; Hb: Haemoglobin; IPTp: Intermittent preventive therapy during pregnancy; IRS: Indoor residual spraying; ITNs: Insecticide-treated nets; LGAs: Local government areas; LLINs: Long-lasting insecticide-treated nets; LRR: Lower River Region; MIS: Malaria Indicator Survey; MoHSW: Ministry of Health and Social Welfare; MRC: Medical Research Council; NBER: North Bank East Region; NBWR: North Bank West Region; NMCP: National Malaria Control Programme; OR: Odds ratio; RBM: Roll-Back Malaria; RDTs: Rapid diagnostic tests; SES: Socio-economic status; SPSS: Statistical Package for the Social Sciences; TWG: Technical working group; URR: Upper River Region; WHO: World Health Organization; WR: Western Region.
}

\section{Competing interests}

The authors declare that they have no competing interests.

\section{Authors' contributions}

STS conceived, designed and coordinated the research project and wrote the manuscript. MJ produced the GIS malaria prevalence maps and also contributed to the manuscript drafting. JJ performed the statistical data analysis and contributed to the interpretation of the results. $\sqcup$ contributed to the drafting of the manuscript and in the literature review. UD provided scientific advice and guidance to the research project and reviewed the draft manuscript. AC participated in the statistical data analysis and in the interpretation of the results. MKB and AS contributed to the literature review and the draft manuscript. All the authors read and approved the final manuscript.

\section{Acknowledgements}

We are grateful to Professor Rachael Dixey, Leeds Metropolitan University, UK and to Professor Johanne Sundby, University of Oslo, Norway, for their useful comments on the initial draft. We acknowledge the comments of $\mathrm{Mr}$ Momodou K Cham, CIAM, Dr Sharmila Lareef-Jah, WHO Country Office, The Gambia; and Ms Lindsey Thatcher, Peace Corps Volunteer, NMCP. We are most grateful to Mr Fodeh Baldeh, Publisher and former Senior Lecturer, University of The Gambia, for the excellent editorial work.

\section{Author details}

${ }^{1}$ National Malaria Control Programme, Kanifing Institutional Layout, Plot \# 17, Kanifing, The Gambia. ${ }^{2}$ Center for International Earth Science Information Network (CIESIN), Columbia University, New York, USA. ${ }^{3}$ Medical Research Council Laboratories, Fajara, Banjul, The Gambia. ${ }^{4}$ Islamic Development Bank, Jeddah, Kingdom of Saudi Arabia. ${ }^{5}$ Gambia Bureau of Statistics, Kanifing Institutional Layout, Kanifing, The Gambia.

Received: 5 July 2014 Accepted: 27 September 2014

Published: 21 November 2014

\section{References}

1. WHO: World Malaria Report 2013. Geneva: World Health Organization; 2013.

2. WHO: World Malaria Report 2012. Geneva: World Health Organization; 2012.

3. Gallup JL, Sachs JD: The Economic Burden of Malaria. Boston, USA: Center for International Development Working Paper No. 52, Harvard University; 2000.

4. Ricci F: Social implications of malaria and their relationships with poverty. Mediterr J Hematol Infect Dis 2012, 4:e2012048.

5. Teklehaimanot A, Mejia P: Malaria and poverty. Ann N Y Acad Sci 2008, 1136:32-37

6. Sachs J, Malaney P: The economic and social burden of malaria. Nature 2002, 415:68-685.

7. de Castro MC, Fisher MG: Is malaria illness among young children a cause or a consequence of low socioeconomic status? Evidence from the United Republic of Tanzania. Malar J 2012, 11:161.

8. Sharma VP: Malaria and poverty in India. Curr Sci 2003, 84:513-515.

9. Tusting LS, Willey B, Lucas H, Thompson J, Kafy HT, Smith R, Lindsay SW: Socioeconomic development as an intervention against malaria: a systematic review and meta-analysis. Lancet 2013, 382:963-972.

10. Goodman C, Hanson K, Mills A, Wiseman V, Worrall E: The Economics of Malaria and its Control. Geneva, Switzerland: WHO/TDR Scientific Working Group on Malaria; 2003:24-27.

11. Gwatkin D, Guillot M: The Burden of Disease among the Global Poor: Current Situations, Future Trends and Implications for Strategy. Washington D.C: World Bank; 2000.

12. Worrall $E$, Basu $S$, Hanson $K$ : Is malaria a disease of poverty? A review of the literature. Trop Med Int Health 2005, 10:1047-1059.

13. Filmer D: Fever and its Treatment among the More and Less Poor in SubSaharan Africa, World Bank Policy Research Working Paper. Washington, D.C: DECRG, The World Bank; 2002.

14. Boadu K, Trovato F: Association of social class with malaria prevalence among household heads in Ghana. Can Stud Popul 2006, 33:271-299.

15. Somi MF, Butler JR, Vahid F, Njau JD, Kachur SP, Abdulla S: Use of proxy measures in estimating socio-economic inequalities in malaria prevalence. Trop Med Int Health 2008, 13:354-364.

16. Onwujekwe O, Uzochukwu B, Dike N, Okoli C, Eze S, Chukwuogo O: Are there geographic and socio-economic differences in incidence, burden and prevention of malaria? a study in southeast Nigeria. Int J Equity Health 2009, 8:45.

17. CHESTRAD: Malaria, Poverty and Health. Ibadan, Nigeria: Center for Health Services Training, Research and Development International; 2000. 
18. Government of The Gambia: The Gambia Malaria Indicator Survey 2010/11. Banjul: Ministry of Health and Social Welfare; 2012

19. Government of The Gambia: The Gambia Multiple Indicator Cluster Survey 2005/2006 Report. Banjul: Gambia Bureau of Statistics; 2007.

20. Government of The Gambia: Housing and Household Characteristics. In 2003 Population and Housing Census. 6th edition. Banjul: Gambia Bureau of Statistics; 2006.

21. Macro ORC: Guidelines for Sampling for the Malaria Indicator Survey. Maryland, USA: Calverton; 2005.

22. Government of The Gambia: The Gambia 2013 Population and Housing Census Preliminary Results. Banjul, The Gambia: Gambia Bureau of Statistics; 2014.

23. Government of The Gambia: Integrated Household Survey, Income and Expenditure Poverty Assessment, 2010. Banjul, The Gambia: Gambia Bureau of Statistics; 2010.

24. Filmer D, Pritchett L: Estimating wealth effects without expenditure data -or tears: an application to educational enrolments' in States of India. Demography 2001, 38:115-132.

25. Verma $V$, Betti $G$ : Taylor linearization sampling errors and design effects for poverty measures and other complex statistics. J App/ Stat 2010, 38:1549-1576.

26. De Beaudrap P, Nabasumba C, Grandesso F, Turyakira E, Schramm B, Boum $Y$ 2nd, Etard JF: Heterogeneous decrease in malaria prevalence in children over a six-year period in south-western Uganda. Malar J 2011, 10:132.

27. Gahutu JB, Steininger C, Shyirambere C, Zeile I, Cwinya-Ay N, Danquah I, Larsen CH, Eggelte TA, Uwimana A, Karema C, Musemakweri A, Harms G, Mockenhaupt FP: Prevalence and risk factors of malaria among children in southern highland Rwanda. Malar J 2011, 10:134

28. Winskill P, Rowland M, Mtove G, Malima RC, Kirby MJ: Malaria risk factors in north-east Tanzania. Malar J 2011, 10:98.

29. Ayele DG, Zewotir T, Mwambi HG: Prevalence and risk factors of malaria in Ethiopia. Malar J 2012, 11:195.

30. Ayele DG, Zewotir TT, Mwambi HG: Semiparametric models for malaria rapid diagnosis test result. BMC Public Health 2014, 14:31.

31. Clarke SE, Bogh C, Brown RC, Pinder M, Walraven GE, Lindsay SW: Do untreated bednets protect against malaria? Trans $R$ Soc Trop Med Hyg 2001, 95:457-462.

32. Somi MF, Butler JRG, Vahid F, Njau JD, Kachur SP, Abdulla S: Is there evidence for dual causation between malaria and socio-economic status? Findings from rural Tanzania. Am J Trop Med Hyg 2007, 77:1020-1027.

33. Government of The Gambia: Integrated Household Survey, Income and Expenditure Poverty Assessment, 2003. Banjul, The Gambia: Gambia Bureau of Statistics; 2005

34. ljumba JN, Lindsay SW: Impact of irrigation on malaria in Africa: paddies paradox. Med Vet Entomol 2001, 15:1-11.

35. Jarju LBS, Fillinger U, Green C, Louca V, Majambere S, Lindsay SW: Agriculture and the promotion of insect pests: swamp rice cultivation and malaria vectors in The Gambia. Malar J 2009, 8:170

36. Yeshiwondim AK, Gopal S, Hailemariam AT, Dengela DO, Patel HP: Spatial analysis of malaria incidence at the village level in areas with unstable transmission in Ethiopia. Int J Health Geogr 2009, 8:5.

37. Kirby MJ, Ameh D, Bottomley C, Green C, Jawara M, Milligan PJ, Snell PC, Conway DJ, Lindsay SW: Effect of two different house screening interventions on exposure to malaria vectors and on anaemia in children in The Gambia: a randomised controlled trial. Lancet 2009, 374:998-1009.

38. Temu EA, Coleman M, Abilio AP, Kleinschmidt I: High prevalence of malaria in Zambezia, Mozambique: the protective effect of IRS versus increased risks due to pig-keeping and house construction. PLOS One 2012, 7:e31409.

39. Ghebreyesus TA, Haile M, Winen KH, Getachew A, Yohannes M, Lindsay SW, Byass P: Household risk factors for malaria among children in the Ethiopian highlands. Trans R Soc Trop Med Hyg 2000, 91:17-21.

40. Sintasath DM, Ghebremeskel T, Lynch M, Kleinau E, Bretas G, Shililu J, Brantly E, Graves PM, Beier JC: Malaria prevalence and associated risk factors in Eritrea. Trans R Soc Trop Med Hyg 2005, 72:682-687.

41. Hiscox A, Khammanithong P, Kaul S, Sananikhom P, Luthi R, Hill N, Brey PT, Lindsay SW: Risk factors for mosquito house entry in the Lao PDR. PLOS One 2013, 8:e62769.
42. Worrall E, Basu S, Hanson K: The Relationship Between Socio-Economic Status and Malaria: A Review of the Literature. In Background Seminar Paper on "Ensuring that Malaria Control Interventions Reach the Poor". London School of Hygiene and Tropical Medicine and The World Bank; 2003.

43. Bousema T, Drakely C, Gesase S, Hashim R, Magesa S, Mosha F, Otieno S, Carneiro I, Cox J, Msuya E, Kleinschmidt I, Maxwell C, Greenwood B, Riley E, Sauerwein R, Chandramohan D, Gosling R: Identification of hot spots of malaria transmission for targeted malaria control. J Infect Dis 2010, 201:1764-1774.

44. Government of The Gambia: The Gambia Multiple Indicator Cluster Survey 2005/2006 Report. Banjul: Gambia Bureau of Statistics; 2012.

45. Ministry of Health and Social Welfare: National Malaria Strategic Plan 2014 2020. Banjul: National Malaria Control Programme; 2013.

46. Ministry of Health and Social Welfare: National Malaria Policy 2014-2020, A Malaria-Free Gambia. Banjul: National Malaria Control Programme; 2013.

47. Imbahale SS, Mukabana WR, Orindi B, Githeko AK, Takken W: Variation in malaria transmission dynamics in three different sites in Western Kenya. J Trop Med 2012, 2012:912408.

48. Bousema T, Griffin JT, Sauerwein RW, Smith DL, Churcher TS, Takken W, Ghani A, Drakeley C, Gosling R: Hitting hotspots: spatial targeting of malaria for control and elimination. PLoS Med 2012, 9:e1001165.

doi:10.1186/1475-2875-13-449

Cite this article as: Sonko et al:: Does socio-economic status explain the differentials in malaria parasite prevalence? Evidence from The Gambia. Malaria Journal 2014 13:449.

\section{Submit your next manuscript to BioMed Central and take full advantage of:}

- Convenient online submission

- Thorough peer review

- No space constraints or color figure charges

- Immediate publication on acceptance

- Inclusion in PubMed, CAS, Scopus and Google Scholar

- Research which is freely available for redistribution 